\title{
Optimization of Deposition Parameters for Thin Silicon Films on Flexible Substrates in a Hot-Wire Chemical Vapor Deposition Reactor
}

\author{
P. Alpuim ${ }^{a}$, M. Ribeiro ${ }^{b}$, S. Filonovich ${ }^{c}$ \\ University of Minho, GCEP - Center of Physics, 4800-058 Guimarães, Portugal \\ apalpuim@fisica.uminho.pt, ${ }^{b}$ miquelbruno@mail.pt, ${ }^{{ }^{c} \text { filan@ fisica.uminho.pt }}$
}

Keywords: nanocrystalline silicon, hot-wire CVD, flexible electronics, thin-film solar cell

\begin{abstract}
This paper studies the deposition of thin silicon films from silane on plastic substrates in a recently build hot-wire chemical vapor deposition reactor. Hydrogen dilution of silane was used to induce amorphous-to-nanocrystalline phase transition. Thin-film deposition rate, $r_{\mathrm{d}}$, is roughly proportional to silane concentration during deposition but the proportionality factor depends on filament temperature, $T_{\text {fil }}$. At $T_{\text {fil }} \sim 2500^{\circ} \mathrm{C}\left(1900^{\circ} \mathrm{C}\right), r_{\mathrm{d}}$ increases from $2.1 \AA / \mathrm{s}(1.2 \AA / \mathrm{s})$ at $97 \% \mathrm{H}_{2}$ dilution to $14.5 \AA / \mathrm{s}(10.7 \AA / \mathrm{s})$ for films deposited from pure silane. At $T_{\text {fil }} \sim 2500^{\circ} \mathrm{C}$, films deposited under $80 \% \mathrm{H}_{2}$ dilution were amorphous, under $90 \% \mathrm{H}_{2}$ dilution the crystalline fraction was $X_{\mathrm{C}}=49.4 \%$ and under $95 \% \mathrm{H}_{2}$ dilution, $X_{\mathrm{C}}=52.8 \%$. At $T_{\text {fil }} \sim 1900^{\circ} \mathrm{C}$, samples were amorphous up to $\sim 95 \% \mathrm{H}_{2}$ dilution where a crystalline fraction of $22.3 \%$ was measured.

Films with amorphous structure have $\sigma_{\mathrm{d}} \sim 10^{-10}-10^{-9} \Omega^{-1} \mathrm{~cm}^{-1}$ while those with a measured crystalline fraction have $\sigma_{\mathrm{d}} \sim 1010^{-5} \Omega^{-1} \mathrm{~cm}^{-1}$, depending on the amount of crystalline fraction and grain size. Films with lower $\sigma_{\mathrm{d}}$ have optical band gap in the range $\sim 1.85-1.9 \mathrm{eV}$, typical of hydrogenated amorphous silicon, while those with higher $\sigma_{\mathrm{d}}$ have larger optical band gap $(\sim 2 \mathrm{eV})$, typical of hydrogenated nanocrystalline silicon.

Adhesion of the films to the plastic substrate was good, as they survived bending to small radius of curvature $(<1 \mathrm{~mm})$ without peeling. Structural, optical and transport properties were similar on films deposited both on PEN and on glass under the same deposition conditions.
\end{abstract}

\section{Introduction}

Until recently the choice of the substrates to use in macroelectronic applications (photocopiers, flat-panel displays, light-sensors or solar cells) has been largely determined by their ability to survive to the high deposition temperatures $\left(>250^{\circ} \mathrm{C}\right)$ generally requested to deposit the thin semiconductor films that make the devices $[1,2]$. This constraint severely reduces the number of candidate materials for substrate, with most common choices typically falling on glass or stainless steel. Since electronic devices bear the mechanical properties of the substrate that they use, these devices are normally brittle and/or rigid [3]. However, the use of new deposition techniques, like hot-wire chemical vapor depostion (HW-CVD), or hydrogen dilution of the reactant gases, is allowing CVD deposition of thin silicon-based films of high electronic quality at much lower substrate temperatures $[4,5,6,7]$. This opens the possibility to use novel types of substrates which in turn will lead to new approaches to conventional applications - like roll-to-roll manufactured flexible solar cells - or to entirely new areas of technology - like sensitive skin [8].

In this paper a recently re-build HW-CVD reactor is used to deposit hydrogenated amorphous silicon (a-Si:H) and hydrogenated nanocrystalline silicon (nc-Si:H) from $\mathrm{SiH}_{4}$ on polyethylene naphthalate (PEN) plastic substrates at a substrate temperature of $T_{\text {sub }}=150^{\circ} \mathrm{C}$. Special attention is devoted to the range of $\mathrm{H}_{2}$-dilution of silane which leads to the amorphous-to-nanocrystalline 
phase transition. A series of samples deposited at different $\mathrm{H}_{2}$ dilutions was prepared by standard 13.56 MHz radio-frequency plasma-enhanced chemical vapor deposition (rf-PECVD) in a twin chamber of the same reactor, for comparison. The best films resulting from this work will be used as the intrinsic layer on n-i-p solar cells fabricated on plastic or in tandem solar cells with two absorbing layers of a-Si:H and nc-Si:H.

\section{Experiment}

Film Preparation. The films were deposited by HW-CVD (HW) and rf-PECVD (RF) in a UHVquality system with a base pressure $\leq 2 \times 10^{-7}$ Torr. This system was recently build and was used for the first time. The sample was clamped to the grounded upper electrode which was heated to the temperature of deposition, $T_{\text {sub }}=150^{\circ} \mathrm{C}$. This temperature was chosen because it is close but inferior to the maximum working temperature of the plastic, PEN.

For the HW deposition, a single tungsten filament of $0.5 \mathrm{~mm}$ diameter and approximately $14 \mathrm{~cm}$ length was bended to a coil and placed $4 \mathrm{~cm}$ from the substrate, and was resistively heated with an AC power supply. Two filament temperatures, corresponding to filament currents of $\sim 14.5 \mathrm{~A}$ and $\sim 16.5 \mathrm{~A}$, were used. The filament temperature was measured with an optical pyrometer $\left(\mathrm{T}_{\text {fil }} \sim\right.$ $1900^{\circ} \mathrm{C}$ and $2500{ }^{\circ} \mathrm{C}$, respectively) and the pressure was kept constant at $20 \mathrm{mTorr}$. For the RF deposition, the inter-electrode distance was $3 \mathrm{~cm}$, the density of RF power used was $175 \mathrm{~mW} / \mathrm{cm}^{2}$ and the pressure was 100 mTorr. Silane flux, $F_{\mathrm{SiH} 4}$ was kept at $1 \mathrm{sccm}$ and hydrogen flux, $F_{\mathrm{H} 2}$, was varied in order to obtain the desired dilution, defined as $F_{\mathrm{H} 2} /\left(F_{\mathrm{H} 2}+F_{\mathrm{SiH} 4}\right)$.

Film Characterization. The room temperature dark conductivity, $\sigma_{\mathrm{d}}$, was obtained from the $I-V$ characteristics of the sample, measured on coplanar Al contacts. Raman spectra were measured in the backscattering geometry using a Raman microprobe. The power of the incident beam was set below $50 \mathrm{~mW}$ to avoid thermally induced crystallization. For nanocrystalline films, the Raman spectrum around the crystalline silicon transverse optical (TO) peak was deconvoluted into their integrated crystalline Gaussian peak, $I_{\mathrm{c}}\left(\sim 520 \mathrm{~cm}^{-1}\right)$, amorphous Gaussian peak, $I_{\mathrm{a}}\left(\sim 480 \mathrm{~cm}^{-1}\right)$, and intermediate Gaussian peak, $I_{\mathrm{m}}\left(\sim 510 \mathrm{~cm}^{-1}\right)[9,10]$. The crystalline fraction, $X_{\mathrm{c}}$, was calculated from $X_{\mathrm{c}}=\left(I_{\mathrm{c}}+I_{\mathrm{m}}\right) /\left(I_{\mathrm{c}}+I_{\mathrm{m}}+I_{\mathrm{a}}\right)$ [11]. For amorphous films, the bond angle deviation $\Delta \theta$ was related to the full width at half maximum (FWHM) $\Gamma$ of the TO peak centered at $480 \mathrm{~cm}^{-1}$ using the expression: $\Delta \theta=\Gamma / 6-2.5[12]$.

$\mathrm{X}$-ray diffraction peaks were measured using the $\mathrm{Cu} K_{\alpha 1}$ line $(\lambda=1.54056 \AA)$. The samples were measured at grazing incidence $\left(1^{\circ}\right)$ using substrate holder rotation $(15 \mathrm{rpm})$. The crystallite size $d_{\mathrm{X} \text {-ray }}$ was calculated from the Scherrer formula $d_{\mathrm{X} \text {-ray }}=k \lambda /\left(B \cos \theta_{\mathrm{B}}\right)$, where $k \sim 0.9, \lambda$ is the wavelength of the X-ray radiation, $B$ is the FWHM of the peaks (in units of $2 \theta$ ) and $\theta_{\mathrm{B}}$ is the angular position of the peak.

\section{Results and Discussion}

Figure 1 shows deposition rate, $r_{\mathrm{d}}$, as a function of $\mathrm{H}_{2}$ dilution for samples deposited by $\mathrm{HW}$ at $T_{\text {fil }}$ $\sim 2500^{\circ} \mathrm{C}$ (high- $T_{\text {fil }}$ ) and $\sim 1900^{\circ} \mathrm{C}$ (low- $\left.T_{\text {fil }}\right)$. Also shown, for comparison, is $r_{\mathrm{d}}$ of RF samples. $r_{\mathrm{d}}$ is roughly proportional to silane concentration (see insert) but the proportionality factor is higher for the highest filament temperature. At $T_{\text {fil }} \sim 2500^{\circ} \mathrm{C}\left(1900^{\circ} \mathrm{C}\right), r_{\mathrm{d}}$ increases from $2.1 \AA / \mathrm{s}(1.2 \AA / \mathrm{s})$ at $97 \% \mathrm{H}_{2}$ dilution to $14.5 \AA / \mathrm{s}(10.7 \AA / \mathrm{s})$ for films deposited from pure silane. $r_{\mathrm{d}}$ is lower in $\mathrm{RF}\left(r_{\mathrm{d}}\right.$ changes between $0.47 \AA / \mathrm{s}$ at $97 \% \mathrm{H}_{2}$ dilution to $4.3 \AA / \mathrm{s}$ with no dilution of silane) than in HW. 


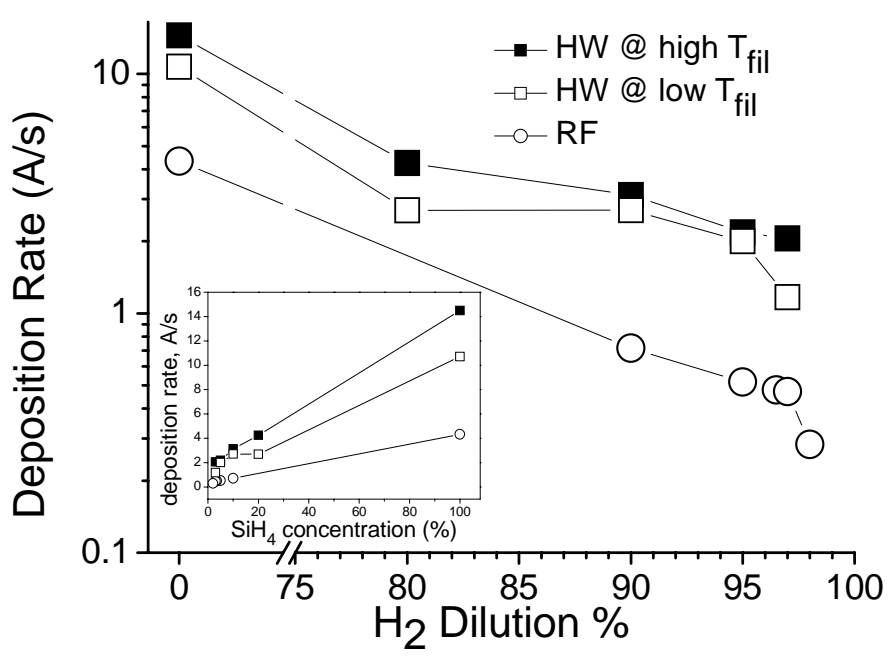

Figure 1. Deposition rate, $r_{\mathrm{d}}$, as a function of $\mathrm{H}_{2}$ dilution for samples deposited by $\mathrm{HW}$ at $\mathrm{T}_{\text {fil }} \sim 2500^{\circ} \mathrm{C}$ (high- $\left.T_{\text {fil }}\right)$ and $\sim 1900^{\circ} \mathrm{C}$ (low- $\left.T_{\text {fil }}\right)$. Also shown, for comparison, is $r_{\mathrm{d}}$ of RF samples. Insert shows same data plotted as a function of silane concentration during film growth. (Lines are guides to the eve.)

have $X_{\mathrm{C}} \neq 0$, while the $80 \%$ diluted sample is amorphous. The average grain size calculated from XRD spectra (not shown) of nanocrystalline samples was $\sim 30 \mathrm{~nm}$.

Figure 3 shows the degree of disorder in the a$\mathrm{Si}: \mathrm{H}$ network grown by HW and RF on PEN and on glass. $\Delta \theta$ measures the average deviation to the tetrahedral Si-bond angle from the FWHM of the $480 \mathrm{~cm}^{-1}$ absorption band of Raman spectrum. This dispersion in the bond angle values, in device quality a-Si:H, is typically $9-10^{\circ}[3]$, which is the same range found in fig.3. Use of $\mathrm{H}_{2}$ dilution reduced bond angle disorder in a-Si:H deposited at $T_{\text {sub }}=150^{\circ} \mathrm{C}$ both in HW and RF (fig.3).

Room-temperature dark conductivity data (fig.4) correlates well with the structural information revealed for the films. Films with amorphous structure have $\sigma_{\mathrm{d}} \sim 10^{-10}-10^{-9} \Omega^{-1} \mathrm{~cm}^{-1}$ while those with crystalline fraction detected by Raman have $\sigma_{\mathrm{d}} \sim 10^{-7}-10^{-5} \Omega^{-1} \mathrm{~cm}^{-1}$, depending on their crystalline fraction and grain size. Figure 4 also shows that $\sigma_{d}$ of samples deposited by HW on plastic is higher for higher $T_{\text {fil }}$, if $\mathrm{H}_{2}$ dilution of
Figure 2 shows the Raman spectra of selected samples deposited by HW at high- $T_{\text {fil }}$ on b) PEN and c) glass. a) shows the Raman spectrum of PEN in the same range of wavenumbers. Samples deposited on glass [c)] are nanocrystalline $\left(X_{\mathrm{C}}=49.4 \%\right.$ at $90 \% \mathrm{H}_{2}$ dilution and $52.8 \%$ at $95 \%$ dilution). Since the Raman spectrum of PEN [a)] has a peak that overlaps the crystalline $\mathrm{Si}$ peak at $520 \mathrm{~cm}^{-1}$, Raman spectra of films on PEN are difficult to study. Nevertheless, comparison with Raman spectra of films on glass [c)] and correlation with optoelectronic properties of the samples leads to the conclusion that only the $90 \%$ and $95 \%$ films on PEN

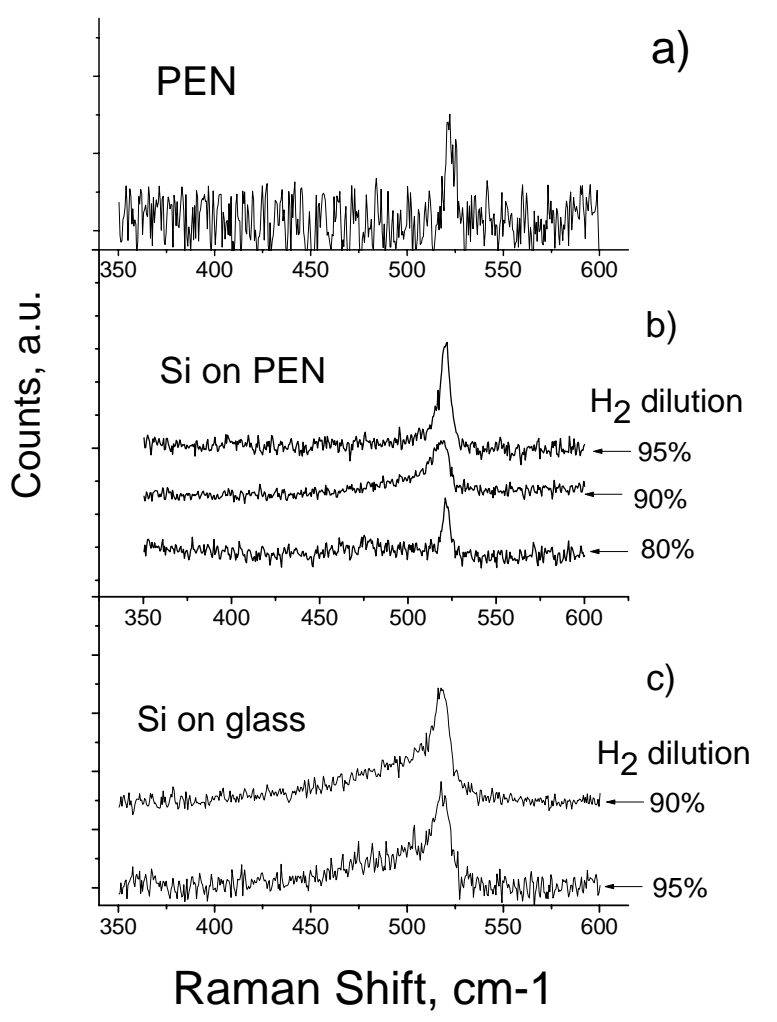

Figure 2. Raman spectra of selected samples deposited by HW-CVD at high- $T_{\text {fil }}$ b) on PEN and c) on glass. a) shows the Raman spectrum of PEN in the same range of wavenumbers. silane is used. This is probably due to a higher efficiency to break the $\mathrm{H}_{2}$ molecule by the hotter filament, leading to a richer atmosphere of atomic hydrogen during film growth when $T_{\text {fil }} \sim 2500^{\circ} \mathrm{C}$ is used than at $T_{\text {fil }} \sim 1900^{\circ} \mathrm{C}$. Two consequences follow: first, high- $T_{\text {fil }}$ deposited amorphous films have higher electronic quality due to a higher degree of passivation of dangling bonds by hydrogen; second: the amorphous-to-nanocrystalline transition occurs at lower $\mathrm{H}_{2}$ dilution $(\sim 90 \%)$ 
at high- $T_{\text {fil }}$ than at low- $T_{\text {fil }}$ where a higher fraction of $\mathrm{H}_{2}$ is required to compensate for the lower efficiency of atomic hydrogen generation.

a-Si:H films on PEN grown by RF could have suffered plasma damage to some extent, judging from the $\sim 2$ orders of magnitude lower $\sigma_{\mathrm{d}}$ measured on these films when compared to corresponding films by RF deposited on glass. This is in contrast with HW-deposited samples where similar films deposited on both types of substrates had similar values of $\sigma_{\mathrm{d}}$, implying that radiation from hot $\mathrm{W}$-filament (up to $\sim 12 \mathrm{~min}$ deposition time) did less or no harm to the plastic. After a 12 minute deposition some degree of permanent deformation of the plastic, due to heating above its glass transition temperature, was observed. For this reason, all HW films had a deposition time limit of $12 \mathrm{~min}$.

Figure 5 shows a Tauc plot where $\sqrt{ } \alpha E$ ( $\alpha$ is the absorption coefficient calculated from transmission data) is plotted as a function of incident photon energy, $E$. The values of the extrapolated optical bandgap obtained from Tauc plots, as illustrated in fig.5, are shown in figure 6 .

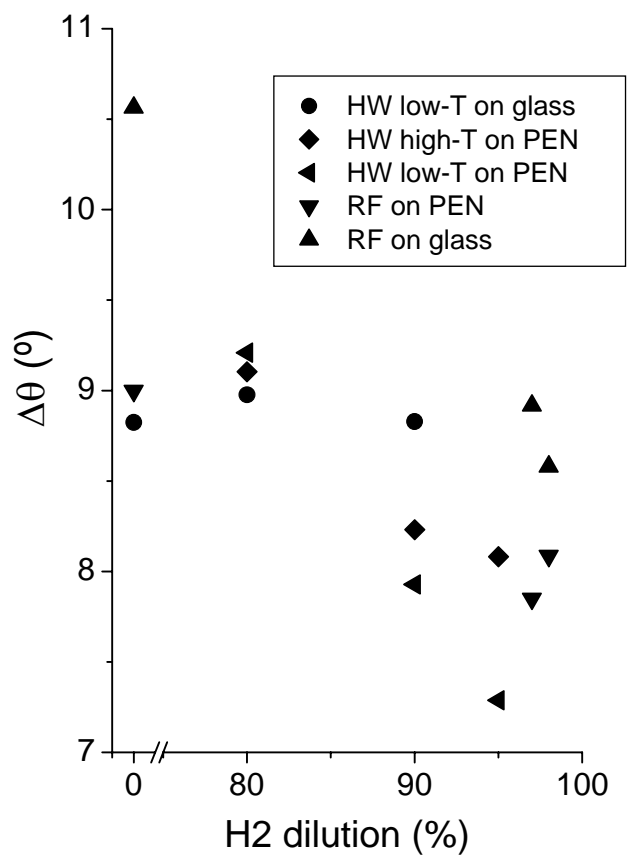

Figure 3. Degree of disorder in a-Si:H tissue grown by $\mathrm{HW}$ and RF on PEN and on glass. $\Delta \theta$ measures average deviation to the tetrahedral Si-bond angle.

It can be seen that, independently of the deposition technique or the parameters used for deposition, samples which were amorphous to Raman and had $\sigma_{\mathrm{d}}$ typical of a-Si:H have optical band gap in the range $\sim 1.85-1.9 \mathrm{eV}$. At extreme $\mathrm{H}_{2}$ dilutions, values of $\mathrm{E}_{\mathrm{opt}}$ become larger, possibly reflecting the formation of an inter-granular porous structure with a high hydrogen concentration.

Several samples deposited on PEN, both at low and high $\mathrm{H}_{2}$ dilution, were bent to a small radius of curvature $(0.75 \mathrm{~mm})$ to test adhesion. All the samples survived this test without peeling.

\begin{tabular}{ccccccc}
$\begin{array}{r}\text { Sam- } \\
\text { ple }\end{array}$ & $\begin{array}{c}T_{\text {Fil }} \\
\left({ }^{\circ} \mathrm{C}\right)\end{array}$ & $\begin{array}{c}\mathrm{H}_{2} \text { dil. } \\
(\%)\end{array}$ & $\begin{array}{c}r_{\mathrm{d}} \\
(\AA / \mathrm{s})\end{array}$ & $\begin{array}{c}E_{\mathrm{opt}} \\
(\mathrm{eV})\end{array}$ & $\begin{array}{c}\sigma_{\mathrm{d}} \\
\left(\Omega^{-1} \mathrm{~cm}^{-1}\right)\end{array}$ & $\begin{array}{c}\% X_{\mathrm{C}} \\
(\text { Raman })\end{array}$ \\
\hline \hline $\mathrm{S} 14$ & $\sim 1900$ & 0 & 10.7 & 1.85 & $2.69 \times 10^{-10}$ & \\
$\mathrm{~S} 15$ & $\sim 1900$ & 80 & 2.7 & 1.87 & $8.20 \times 10^{-12}$ & \\
$\mathrm{~S} 17$ & $\sim 1900$ & 90 & 2.7 & 1.94 & $6.54 \times 10^{-10}$ & \\
$\mathrm{~S} 18$ & $\sim 1900$ & 95 & 2.2 & 1.94 & $4.82 \times 10^{-9}$ & 22.4 \\
$\mathrm{~S} 21$ & $\sim 1900$ & 97 & 1.2 & 2.44 & - & \\
$\mathrm{S} 9$ & $\sim 2500$ & 0 & 14.5 & 1.91 & $5.77 \times 10^{-10}$ & \\
$\mathrm{~S} 10$ & $\sim 2500$ & 80 & 4.3 & 1.69 & $1.66 \times 10^{-8}$ & \\
$\mathrm{~S} 11$ & $\sim 2500$ & 90 & 3.1 & 1.90 & $9.37 \times 10^{-7}$ & 49.4 \\
$\mathrm{~S} 13$ & $\sim 2500$ & 95 & 2.2 & 1.94 & $1.84 \times 10^{-7}$ & 52.8 \\
$\mathrm{~S} 20$ & $\sim 2500$ & 97 & 2.1 & 2.19 & $7.33 \times 10^{-6}$ & \\
\hline \hline
\end{tabular}

Table I. Structural, optical and transport properties of samples deposited by HW-CVD on plastic (PEN) at $T_{\text {sub }}=150^{\circ} \mathrm{C}$.

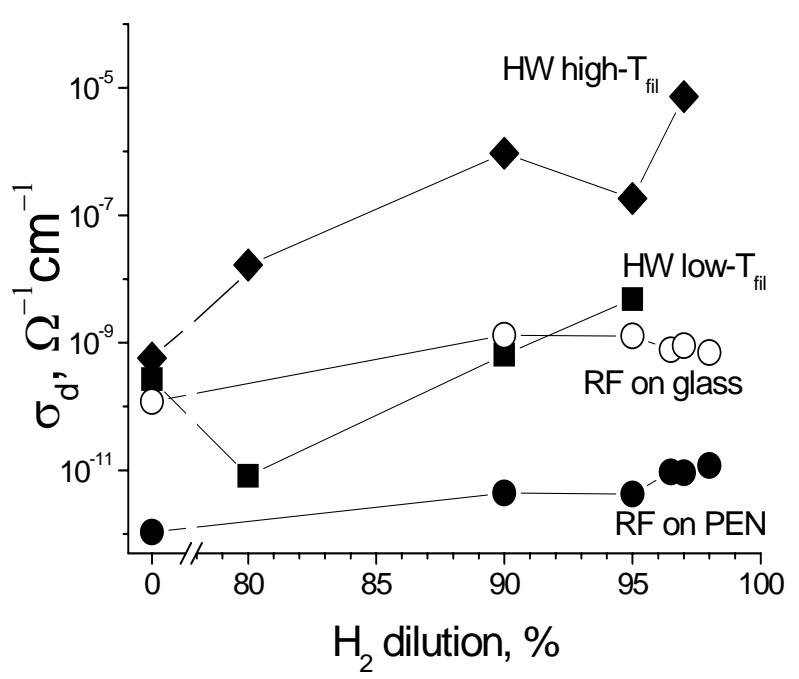

Figure 4 Room-temperature dark conductivity, $\sigma_{\mathrm{d}}$, of samples deposited on PEN (solid symbols) by HW (squares and diamonds) and by RF (circles). Also shown are $\sigma_{\mathrm{d}}$ of RF samples deposited on glass (open circles). Lines are guides to the eye. 


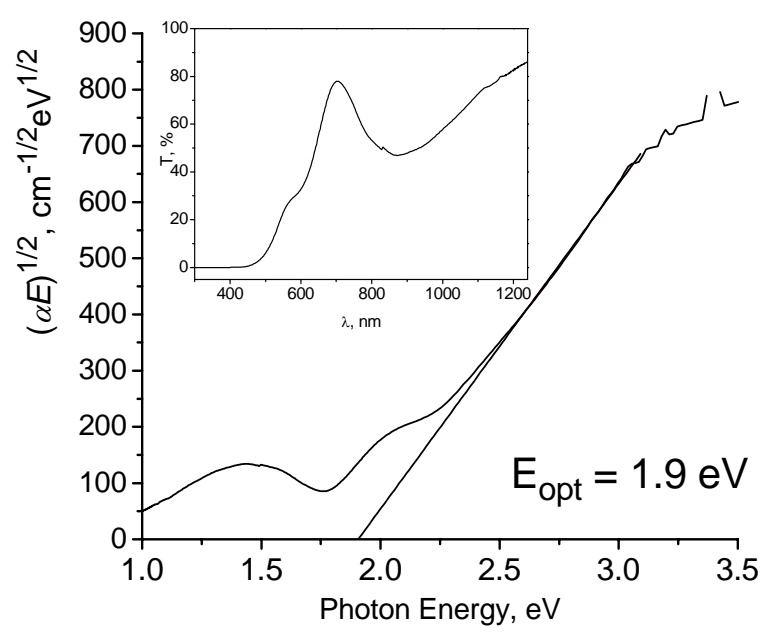

Figure 5 Tauc plot for sample \#9, deposited by HW from pure $\mathrm{SiH} 4$ (on inset - transmission spectrum

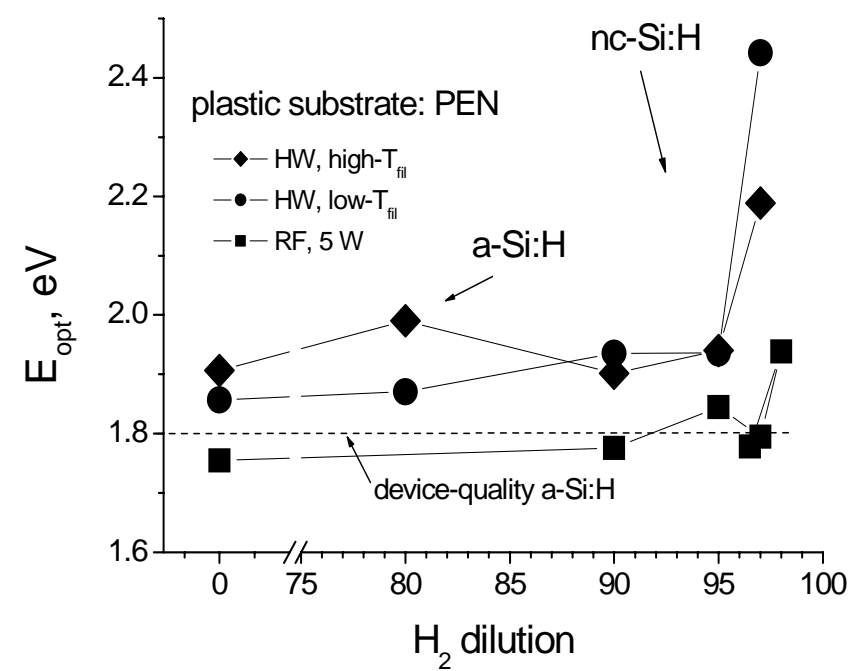

Figure 6 Optical band gap, $E_{\text {opt }}$, calculated from Tauc plot of Si films deposited on PEN.

\section{Conclusions}

nc-Si:H silicon thin films can be prepared on plastic substrates by HW-CVD at a substrate temperature of $150^{\circ} \mathrm{C}$, using $\mathrm{H}_{2}$ dilution of silane, at different filament temperatures. For a lower $T_{\text {fil }}\left(\sim 1900^{\circ} \mathrm{C}\right)$ the $\mathrm{H}_{2}$ dilution necessary to induce the amorphous-to-nanocrystalline phase transition is higher $\left(\sim 95 \% \mathrm{H}_{2}\right.$ dilution) than at a higher $T_{\text {fil }}\left(\sim 2500^{\circ} \mathrm{C}\right)$, where a $90 \% \mathrm{H}_{2}$-diluted sample was nanocrystalline. For dilutions lower than these thresholds, samples are amorphous at both $T_{\text {fil }}$. The deposition rate of films scales approx. linearly with silane concentration in the reactor and is 3-5 times higher in HW than in rf-PECVD, with the particular choice of deposition parameters made in this work. Films deposited by HW show similar structural, transport and optical properties irrespective of the choice of PEN or glass substrate. Both a-Si:H and nc-Si:H films grown on plastic are highly adherent to the substrate and can survive bending to very small radius $(<1 \mathrm{~mm})$ of curvature without peeling.

One of the authors (S.F.) acknowledges FCT for his post-doc grant. The authors acknowledge Physics Department of University of Aveiro for Raman measurements.

\section{References}

[1] C. C. Tsai, Amorphous Silicon and Related Materials, edited by Hellmut Fritzsche (World Scientific Publishing Company, 1988) pp.123-147.

[2] A. Matsuda, J.Non-Cryst. Solids Vol. 59-60 (1983), p. 767.

[3] R. A. Street, Amorphous Silicon Electronics in MRS Bulletin, November 1992, p.70.

[4] P. Alpuim, V. Chu and J. P. Conde, J. Appl. Phys. Vol. 86 (1999), p.3812.

[5] P. Roca i Cabarrocas, Appl. Phys. Lett. Vol. 65 (1994), p. 1674.

[6] E. Srinivasan, D. A. Lloyd, G. N. Parsons, J.Vac.Sci.Technol.A Vol. 15(1) (1997), p.77.

[7] M. Yamaguchi, K. Morigaki, Phil. Mag. B Vol. 79 (3) (1999), p.87.

[8] V. Lumelsky, M. S. Shur, S. Wagner, IEEE Sensors Jour. Vol. 1 (2001), p.1.

[9] T. Kaneko, M. Wakagi, K. Onisawa, T. Minemura, Appl. Phys. Lett Vol. 64 (1994), p.1865.

[10] Veprek, F. A. Sarott, Z. Iqbal, Phys. Rev.B Vol. 36 (1987), p.3344.

[11] Y. He, C. Ying, G. Cheng, L. Wang, X. Liu, G. Y. Hu, J. Appl. Phys. Vol. 75 (1994), p.797.

[12] Y. Hiroyama, R. Suzuki, Y. Hirano, F. Sato, T. Motooka, Jpn. J. Appl. Phys., Part1, Vol.34 
(1995), p.5515. 